\title{
Empoderamiento y apoyo social en pacientes con enfermedad renal crónica: estudio de caso en Michoacán, México
}

\author{
María Fernanda Carrillo Vega, ${ }^{1}$ Blanca Estela Pelcastre Villafuerte, ${ }^{2}$ Guillermo \\ Salinas Escudero, ${ }^{3}$ Luis Durán Arenas ${ }^{4}$ y Malaquías López Cervantes ${ }^{5}$
}

Forma de citar Carrillo Vega MF, Pelcastre Villafuerte BE, Salinas Escudero G, Durán Arenas L, López Cervantes M. Empoderamiento y apoyo social en pacientes con enfermedad renal crónica: estudio de caso en Michoacán, México. Rev Panam Salud Publica. 2017;41:e164. doi: 10.26633/RPSP.2017.164

RESUMEN Objetivo. Identificar el nivel de empoderamiento y las características del apoyo social de los individuos con enfermedad renal crónica (ERC).

Métodos. Estudio transversal en personas con enfermedad renal crónica que habitan en el municipio de Hidalgo, Michoacán, México, y que asisten a la Asociación de Enfermos del Riñón, Asociación Civil (A.C.). Se indagó sobre el perfil sociodemográfico y las características personales asociadas a la enfermedad, el nivel de empoderamiento, el nivel de apoyo social y el funcionamiento familiar.

Resultados. Cerca de $90 \%$ de la muestra cuenta con apoyo social suficiente, mientras que $40 \%$ de los participantes pertenece a familias semirrelacionadas o relacionadas. El puntaje de empoderamiento global fue de 117,5 $\pm 14,3$; el empoderamiento comunitario fue más alto en el grupo de mayor edad $(P<0,05)$. La interacción social positiva es el componente del apoyo social que correlaciona con mayor fuerza con el nivel de empoderamiento $(r=0,333 ; P<0,01)$.

Conclusiones. El empoderamiento está determinado por, y es un determinante del apoyo social y ha facilitado el acceso a la terapia de reemplazo renal en esta comunidad.

Palabras clave Poder; apoyo social; insuficiencia renal crónica.

La enfermedad renal crónica (ERC) es un problema de salud pública a nivel mundial con una prevalencia estimada

\footnotetext{
Departamento de Investigación de Epidemiología Clínica, Instituto Nacional de Geriatría, Ciudad de México, México.

2 Centro de Investigación en Sistemas de Salud, Instituto Nacional de Salud Pública, Cuernavaca, México.

3 Centro de Estudios Económicos y Sociales en Salud, Hospital Infantil de México Federico Gómez, Ciudad de México, México.

4 Centro Nacional para la Salud de la Infancia y la Adolescencia, Ciudad de México, México.

5 Dirección General de Planeación y Desarrollo en Salud, Ciudad de México, México. Enviar correspondencia a Malaquías López Cervantes, autor. correspondiente.1401@gmail.com
}

de 13\% (1). En México, las cifras exactas se desconocen debido a la carencia de un sistema nacional de registro; sin embargo, se han reportado prevalencias que oscilan entre $7 \%$ y $33 \%$ (2). Estas cifras han motivado el interés en el estudio de sus determinantes y su impacto, con el reporte de que, en muchas ocasiones, el retardo en su detección y tratamiento es una constante que afecta a los grupos más vulnerables. Al respecto, se ha reportado que la prevalencia de la ERC en poblaciones de muy bajos recursos en el territorio mexicano es de dos a tres veces mayor en comparación con la población general, y que se desconoce la causa de la enfermedad en aproximadamente $30 \%$ de los casos (3). Esto es reflejo de un sistema de salud carente de capacidad para responder a las necesidades de la población, en el cual el acceso a la atención se encuentra determinado por la afiliación a las instituciones de seguridad social, situación definida a su vez por la pertenencia al mercado laboral formal, de manera que los menos favorecidos tienen acceso limitado o nulo a los recursos para la atención de la enfermedad (4). Una tasa de mortalidad tres veces mayor en la población no afiliada es

Este es un artículo de acceso abierto distribuido bajo los términos de la licencia Creative Commons Attribution-NonCommercial-NoDerivs 3.0 IGO, que permite su uso, distribución y reproducción en cualquier medio, siempre que el trabajo original se cite de la manera adecuada. No se permiten modificaciones a los artículos ni su uso comercial. Al reproducir un artículo no debe haber ningún indicio de que la OPS o el artículo avalan a una organización o un producto específico. El uso del logo de la OPS no está permitido. Esta leyenda debe conservarse, junto con la URL original del artículo. 
el dato que mejor refleja la gravedad del problema (5).

Por otra parte, quienes están afiliados suelen recibir atención de poca calidad. En ambos casos, los individuos se ven obligados a realizar acciones de diferente naturaleza para acceder a la atención de la enfermedad y así asegurar su supervivencia. Para que estas acciones sean exitosas, los individuos deben contar con ciertas características que los lleven a tener éxito en la tarea.

En este sentido, se ha documentado que una de las herramientas de mayor utilidad en esta búsqueda es el empoderamiento (6), que puede ser impulsado por o generador de apoyo social (7).

El empoderamiento es el proceso de toma de decisiones autónomas por medio del cual el individuo obtiene acceso a los recursos que lo ayudan a gestionar su condición actual y llevar a cabo acciones valiosas y significativas que impactan en su persona y contexto (8). En el ámbito de la salud, el empoderamiento permite al individuo obtener el control sobre su salud y mantener la integración social (9). Para que esto suceda, es necesaria la interacción del individuo con otros sujetos (10), específicamente con la red de relaciones sociales que lo rodea y que es percibida por este como significativa (11). Mediante esta red, el individuo obtendrá apoyo social, mecanismo que sirve como un "factor protector" contra la vulnerabilidad de los individuos frente a los efectos del estrés sobre la salud (12).

Pocos estudios han intentado clarificar la relación existente entre el empoderamiento y el apoyo social. En algunos de ellos (13) se presenta al apoyo social como una de las variables impulsoras del empoderamiento, al ser un recurso que debe controlarse para conseguir los objetivos y metas establecidas. Autores como Rosenfield, en cambio, apuntan hacia el empoderamiento como promotor del apoyo social, pues un individuo empoderado es más capaz de buscar apoyo en diferentes redes en comparación con quienes carecen de la capacidad para tomar el control de sus vidas (14).

El presente estudio tiene como objetivo identificar el nivel de empoderamiento y las características del apoyo social de los individuos con ERC que habitan en una comunidad en la que las acciones para obtener el tratamiento para la ERC se realizan de manera constante y en la que la red de apoyo parece jugar un papel determinante en este empoderamiento.

\section{MATERIALES Y MÉTODOS}

\section{Diseño y población de estudio}

Para alcanzar el objetivo del presente trabajo se llevó a cabo un estudio transversal descriptivo en la Asociación de Enfermos del Riñón A.C. de Ciudad Hidalgo, Municipio de Hidalgo, Michoacán, México.

Este municipio se caracteriza por tener la mayor prevalencia de ERC en el estado. Es esta localidad se han registrado aproximadamente 1000 casos, lo que representa $20 \%$ del total a nivel estatal. En Ciudad Hidalgo, cabecera del municipio, se halla la Asociación de Enfermos del Riñón A.C., cuya actividad se centra en brindar apoyo emocional e instrumental a personas con la enfermedad en sus diferentes etapas, sobre todo a aquellas personas que ya cuentan con terapia de reemplazo renal (TRR). De acuerdo con su censo, cerca del $10 \%$ del total de personas con ERC en el Municipio de Hidalgo (aproximadamente 134 individuos), residen en la cabecera del municipio.

La elección de esta área responde a la necesidad de explorar el tema desde un entorno caracterizado por la presencia de desigualdades en salud, cuya alta prevalencia de ERC y la carencia de recursos para la atención a la enfermedad obligan a las personas que sufren esta enfermedad a tomar el control de los recursos con los que cuentan para poder acceder al tratamiento de la enfermedad. Esta característica parece responder a un proceso de empoderamiento, que ha trascendido en la creación de una de las pocas asociaciones civiles en México destinadas a esta población.

El tamaño de muestra fue calculado mediante la fórmula para la estimación de una proporción en una población, tomando en cuenta que en el censo de la Asociación se tiene registrado un total de 134 personas con ERC y esperando que por lo menos un $23 \%$ de las personas tuvieran un nivel de empoderamiento medio. Considerando los datos reportados por Pick y cols. (15), el tamaño de muestra fue de 69 personas, conseguido mediante muestreo aleatorio simple. Se incluyeron a las personas con diagnóstico confirmado de ERC entre 18 y 85 años de edad, con o sin TRR, que decidieran participar de manera voluntaria y firmaran la carta de consentimiento informado.

\section{Recolección de datos}

Las autoridades de la asociación realizaron contacto directo con los miembros, seleccionados de manera aleatoria, para convocarlos a una reunión informativa, cuya finalidad fue dar a conocer las características del estudio, los objetivos, la metodología, y el tiempo que duraría la aplicación de los cuestionarios. Al finalizar la presentación de la investigadora principal, se realizó una sesión de dudas, tras lo cual se realizaron citas para aplicar los instrumentos.

\section{Identificación del perfil sociodemográfico}

La información se recolectó mediante un cuestionario elaborado ex profeso para el presente estudio. Se incluyeron preguntas acerca de las características individuales y familiares, la afiliación a instituciones o programas de salud, el nivel socioeconómico, los antecedentes patológicos familiares y personales de importancia relacionados con la ERC. Se incluyeron preguntas sobre el tiempo con el diagnóstico con la ERC, el personal de salud que lo diagnosticó y le proporciona tratamiento en la actualidad, el tipo de TRR, el tiempo que lleva con TRR; se incluyeron preguntas para indagar acerca de la recepción de información y capacitación sobre la enfermedad y sus implicaciones.

\section{Nivel de empoderamiento}

El nivel de empoderamiento se determinó mediante la Escala de Agencia Personal y Empoderamiento (ESAGE). Consta de 42 reactivos agrupados en las siguientes variables: autoeficacia, autodeterminación, control sobre mis conductas, pensamiento independiente, identificación de necesidad de cambio, miedo al éxito, reconocimiento de $\mathrm{mi}$ aprendizaje, percepción de mi contexto y control sobre mi entorno. Las cuatro opciones de respuesta ("nunca", "casi nunca", "casi siempre", "siempre") se presentan en escala tipo Likert. Dicha escala fue elaborada y validada en población mexicana (15) y se obtuvieron valores de alfa de Cronbach cercanas a uno (agencia: $\alpha=0,718$; empoderamiento: $\alpha=0,749)$.

Antes de su aplicación, se explicaron los objetivos y características del instrumento, de tal manera que la escala pudiera ser autoaplicada. En aquellos casos en los que el participante no supiera leer o escribir, la escala fue completada con apoyo del encuestador. 


\section{Apoyo social}

El estudio de la red de apoyo social se realizó mediante dos escalas de autoaplicación que, al igual que con la ESAGE, fueron explicadas con detenimiento a los participantes y completadas por el encuestador en caso de que el participante así lo solicitara. Se aplicó la encuesta de apoyo social retomada del Estudio de resultados médicos (MOS, por sus siglas en inglés), para pacientes con enfermedades crónicas (16). Se trata de un cuestionario breve y autoadministrado, que consta de 20 reactivos, cada uno con respuestas en escala Likert de cinco opciones (casi siempre, muchas veces, a veces sí y a veces no, pocas veces, casi nunca) mediante las cuales se evalúa el apoyo social en cuatro dimensiones: emocional e informacional, instrumental, interacción social positiva y apoyo afectivo. Los autores del instrumento reportaron valores de alfa de Chronbach cercanos a uno: apoyo emocional e informacional $(\alpha=0,96)$, apoyo instrumental $(\alpha=0,92)$, interacción positiva $(\alpha=0,94)$, apoyo afectivo $(\alpha=0,91)(16)$. El instrumento se ha validado en varios países de América Latina y se obtuvieron valores muy similares $(17,18)$.

Además, se utilizó la Escala de Evaluación de la Cohesión y Adaptabilidad Familiar (FACES III) $3^{\circ}$ versión $(19,20)$, que ha sido validada en población mexicana obteniendo valores de alfa de Chronbach cercanos a $1(\alpha=0,70)$. La escala original consta de 40 reactivos, cada uno con una escala Likert de cinco opciones (casi siempre, muchas veces, a veces sí y a veces no, pocas veces, casi nunca), divididos en dos partes. Para fines del presente trabajo solo se aplicó la sección que evalúa el nivel de cohesión (familia no relacionada, semirrelacionada, relacionada y aglutinada) y flexibilidad de la familia (rígida, estructurada, flexible y caótica) tal como el sujeto la percibe en ese momento.

A cada participante se le proporcionó la descripción detallada del estudio, tanto en forma verbal como escrita; en esta última se incluyó la descripción del estudio, los objetivos, beneficios y posibles riesgos. Se les solicitó su firma, con la que manifestaron su consentimiento informado. Todos los datos obtenidos se mantendrán como confidenciales.

El presente estudio se considera de mínimo riesgo, debido a que no se utilizaron procedimientos invasivos. Para su realización se siguieron los preceptos de la declaración Helsinki y Reglamento de la Ley General de la Salud en materia de investigación para la salud.

El estudio contó con la aprobación del comité de investigación y ética de la Universidad Nacional Autónoma de México, registro 143/2014.

\section{Análisis estadístico}

La información recabada fue analizada mediante el paquete estadístico STATA v.13.1. Se obtuvieron la media y la desviación estándar para las variables continuas, y frecuencias y porcentajes para las variables categóricas. Se realizó comparación de medias mediante $\mathrm{t}$ de Student y comparación de proporciones por medio de la $x^{2}$ de Pearson. Se determinó la correlación de las variables mediante correlación de Pearson.

\section{RESULTADOS}

Participaron en estudio un total de 69 pacientes, de los cuales $57 \%(n=39)$ era hombre (cuadro 1). La mediana de edad fue de 36 años (intervalo de confianza de $95 \%$ 26,9-47,1); cerca de $71 \%$ de los menores de 36 años era soltero; la misma proporción de casados se observó en los mayores de 36 años. Cerca de $40 \%$ de la población se hallaba en el nivel socioeconómico $\mathrm{D}$, caracterizado porque los inmuebles pueden ser propios, pero con carencia de los principales bienes y servicios satisfactores. Del $32 \%(n=22)$ de individuos que cuenta con seguridad social, el $43 \%(n=15)$ es menor de 38 años.

La media de tiempo con la ERC fue de $5,3 \pm 5,7$ años y con terapia de reemplazo renal de 2,9 $\pm 3,3$ años. La principal TRR fue la hemodiálisis, sobre todo en los mayores de 38 años, mientras que el trasplante fue más frecuente en los más jóvenes. Cerca de $60 \%$ de la muestra, en su mayoría los participantes de menor edad, hace uso de servicios como nutrición y psicología; principalmente dentro de las instalaciones de los servicios de salud, pues la asociación no cuenta con este tipo de servicios.

Se observó que cerca de $90 \%$ de la muestra cuenta con apoyo social suficiente en todas las esferas, con un puntaje medio de 71,4 $\pm 12,8$ (cuadro 2). De los participantes, $36 \%$ pertenece a familias semirrelacionadas o relacionadas y cerca de $60 \%$ pertenece a familias caóticas; no se observaron diferencias significativas por grupo de edad en ninguna de las escalas. El puntaje de empoderamiento global (cuadro 3) fue de 117,5 $\pm 14,3$; no se observaron diferencias por grupo de edad en torno al empoderamiento individual; sin embargo, el empoderamiento comunitario fue más alto en el grupo de mayor edad $(P<0,05)$. Se observó que el locus de control es la dimensión del empoderamiento que muestra diferencias por grupo de edad $(P<0,05)$.

Como se observa en el cuadro 4 , la interacción social positiva es el componente del apoyo social que correlaciona con mayor fuerza con el nivel de empoderamiento $(r=0,333 ; P<0,01)$, seguido por el apoyo emocional $(r=0,245, P<0,05)$.

\section{DISCUSIÓN}

El presente estudio representa uno de los primeros acercamientos al fenómeno del empoderamiento y la red social en pacientes con ERC en el contexto mexicano. Los resultados demuestran que el empoderamiento individual es un proceso presente en los participantes, que trasciende al nivel comunitario principalmente entre los individuos de mayor edad, lo que con base en la teoría de McMillan $(21,22)$ nos lleva a pensar en la presencia de un mayor sentimiento de pertenencia y arraigo en este segmento de la muestra. La red social principal es la familia que, de acuerdo con los participantes, se caracteriza por ser una red carente de roles y de liderazgo. A pesar de esta peculiaridad, el apoyo social recibido es percibido por los individuos como adecuado.

El nivel adecuado de empoderamiento guarda una relación estrecha con la pertenencia a la asociación. En este sentido, el acercamiento a este tipo de grupos de apoyo denota la capacidad del individuo para plantear y lograr objetivos, dimensiones que forman parte del empoderamiento individual. Por otro lado, los individuos que acceden a estos grupos son provistos con herramientas que incrementan capacidades como el autocontrol, la toma de decisiones informada y otras propias del empoderamiento.

Este último constructo ha sido ampliamente estudiado en contextos caracterizados por la presencia constante de desigualdades en la distribución de recursos $(23,24)$. A pesar de que México se caracteriza por ser un país de marcada inequidad (25-27), poco se ha explorado 
CUADRO 1. Características generales de la población por grupo de edada

\begin{tabular}{|c|c|c|c|c|c|c|c|}
\hline & \multicolumn{2}{|c|}{ Total } & \multicolumn{2}{|c|}{$\leq 36$ años } & \multicolumn{2}{|c|}{$>36$ años } & \multirow{2}{*}{$P$} \\
\hline & $\mathrm{n}=69$ & $\%$ & $\mathrm{n}=35$ & $\%$ & $\mathrm{n}=34$ & $\%$ & \\
\hline \multicolumn{8}{|l|}{$\overline{S e x 0}$} \\
\hline Femenino & 30 & 43,5 & 15 & 42,9 & 15 & 44,1 & 0,916 \\
\hline Masculino & 39 & 56,5 & 20 & 57,1 & 19 & 55,9 & \\
\hline \multicolumn{8}{|l|}{ Religión } \\
\hline Católica & 67 & 97,1 & 35 & 1,0 & 32 & 94,1 & 0,145 \\
\hline \multicolumn{8}{|l|}{ Estado civil } \\
\hline Soltero & 29 & 42,7 & 24 & 70,6 & 5 & 14,7 & 0,000 \\
\hline Casado & 33 & 48,5 & 9 & 26,5 & 24 & 70,6 & \\
\hline Viudo & 3 & 4,4 & 0 & 0 & 3 & 8,8 & \\
\hline Unión libre & 3 & 4,4 & 1 & 2,9 & 2 & 5,9 & \\
\hline \multicolumn{8}{|l|}{ Nivel socioeconómico } \\
\hline C & 9 & 13,0 & 5 & 14,3 & 4 & 11,8 & \\
\hline $\mathrm{C}_{+}$ & 1 & 1,5 & 1 & 2,9 & 0 & 0,0 & \\
\hline \multicolumn{8}{|l|}{ Antecedentes familiares de importancia } \\
\hline HTA & 5 & 7,3 & 3 & 8,6 & 2 & 5,9 & 0,962 \\
\hline DM & 16 & 23,2 & 7 & 20 & 9 & 26,5 & \\
\hline HTA+DM & 15 & 21,7 & 8 & 22,9 & 7 & 21,0 & \\
\hline $\mathrm{HTA}+\mathrm{ERC}$ & 2 & 2,9 & 1 & 2,9 & 1 & 2,9 & \\
\hline $\mathrm{DM}+\mathrm{ERC}$ & 10 & 14,5 & 8 & 22,9 & 2 & 5,9 & \\
\hline $\mathrm{HTA}+\mathrm{DM}+\mathrm{ERC}$ & 6 & 8,7 & 3 & 8,6 & 3 & 8,8 & \\
\hline Sin antecedentes & 15 & 21,7 & 5 & 14,3 & 10 & 29,4 & \\
\hline \multicolumn{8}{|l|}{ Diagnósticos relacionados con la ERC } \\
\hline \multicolumn{8}{|l|}{ Lugar donde recibe atención para la ERC } \\
\hline Asociación de enfermos del riñón & 2 & 2,9 & 1 & 2,9 & 1 & 2,9 & \\
\hline Terapia de reemplazo renal (\%) & 38 & 55,2 & 12 & 34,3 & 26 & 76,5 & \\
\hline Hemodiálisis & 13 & 18,8 & 11 & 31,5 & 2 & 5,9 & 0,002 \\
\hline Trasplante & 9 & 13,0 & 6 & 17,1 & 3 & 8,8 & \\
\hline Diálisis peritoneal & 9 & 13,0 & 6 & 17,1 & 3 & 8,8 & \\
\hline Tiempo con terapia de reemplazo renal (años) & \multicolumn{2}{|c|}{$2,9 \pm 3,3$} & \multicolumn{2}{|c|}{$3,7 \pm 3,5$} & \multicolumn{2}{|c|}{$2,3 \pm 2,9$} & 0,107 \\
\hline \multicolumn{8}{|l|}{ Médico que lo atiende en la actualidad } \\
\hline Nefrólogo & 43 & 62,3 & 20 & 57,1 & 23 & 67,7 & 0,659 \\
\hline Médico familiar o general & 17 & 24,6 & 10 & 28,6 & 7 & 20,6 & \\
\hline Otro & 9 & 13,0 & 5 & 14,3 & 4 & 11,8 & \\
\hline Recibe atención o asesoría de otros profesiona & & & & & & & \\
\hline Nutriólogo & 20 & 29,0 & 7 & 20,0 & 13 & 38,2 & 0,279 \\
\hline Nutriólogo + psicólogo & 26 & 37,7 & 14 & 40,0 & 12 & 35,3 & \\
\hline Médico clínico & 2 & 2,9 & 1 & 2,9 & 1 & 2,9 & \\
\hline Psicólogo + médico clínico & 2 & 2,9 & 0 & 0,0 & 2 & 5,9 & \\
\hline Ninguno & 19 & 27,5 & 13 & 37,1 & 6 & 17,7 & \\
\hline Información sobre la ERC (\%) & & & & & & & \\
\hline Se le brinda & 62 & 89,9 & 29 & 82,9 & 33 & 97,1 & 0,052 \\
\hline No se le brinda & 7 & 10,1 & 6 & 17,1 & 1 & 2,9 & \\
\hline
\end{tabular}




\begin{tabular}{|c|c|c|c|c|c|c|c|}
\hline & \multicolumn{2}{|c|}{ Total } & \multicolumn{2}{|c|}{$\leq 36$ años } & \multicolumn{2}{|c|}{$>36$ años } & \multirow{2}{*}{$p$} \\
\hline & $\mathrm{n}=69$ & $\%$ & $\mathrm{n}=35$ & $\%$ & $\mathrm{n}=34$ & $\%$ & \\
\hline \multicolumn{8}{|l|}{ Lugar donde se brinda información (\%) } \\
\hline Hospital de secretaría de salud & 36 & 58,1 & 15 & 51,7 & 21 & 63,6 & 0,687 \\
\hline Hospital o clínica de seguridad social & 14 & 22,6 & 8 & 27,6 & 6 & 18,2 & \\
\hline Hospital o clínica privada & 3 & 4,8 & 1 & 3,5 & 2 & 6,1 & \\
\hline Asociación de enfermos del riñón & 9 & 14,5 & 5 & 17,2 & 4 & 12,1 & \\
\hline Casi siempre & 9 & 14,5 & 5 & 17,2 & 4 & 12,1 & \\
\hline Algunas veces & 5 & 8,1 & 1 & 3,5 & 4 & 12,1 & \\
\hline Casi nunca & 1 & 1,6 & 0 & 0,0 & 1 & 3,0 & \\
\hline
\end{tabular}

a Los valores de $P$ para la comparación por grupos de edad se presentan como promedio \pm desviación estándar; los datos categóricos se presentan como frecuencia (porcentaje). HTA, hipertensión arterial: DM, diabetes mellitus; ERC, enfermedad renal crónica.

Nivel socioeconómico: E, carece de todos los servicios y bienes satisfactores; D, cuenta con una propiedad pero carece de diversos servicios y satisfactores; D+, tiene cubierta la mínima infraestructura sanitaria de su hogar; C-, tiene cubiertas las necesidades de espacio y sanidad y cuenta con los enseres y equipos que le aseguran el mínimo de practicidad y comodidad en el hogar; $\mathrm{C}$, alcanza un nivel de vida práctica y con ciertas comodidades, y cuenta con una infraestructura básica en entretenimiento y tecnología; $\mathrm{C}+$, tiene cubiertas todas las necesidades de calidad de vida, sin embargo tiene ciertas limitantes para invertir y ahorrar para el futuro.

acerca del proceso de empoderamiento y de cómo es posible alcanzarlo a partir de los recursos que la red le proporciona al individuo con el objetivo de acceder al tratamiento de las enfermedades.

De acuerdo con nuestros resultados, la TRR más frecuente entre los individuos de la asociación fue la hemodiálisis. Este dato es difícil de comparar con otros a nivel internacional debido a las diferencias entre los sistemas de salud, y a nivel nacional debido a la carencia de un registro, por tipo de lugar de atención y por comunidad, sea rural o urbana. No obstante, nuestros resultados difieren de los reportados por MéndezDurán y cols. (28) para el Instituto Mexicano del Seguro Social (IMSS) y por la Sociedad Latinoamericana de Nefrología e Hipertensión (29), quienes reportan que la TRR de mayor uso a nivel nacional es la diálisis peritoneal (DP). Podemos atribuir esta diferencia a las características de los servicios de salud que atienden a esta población pues, como se observa, cerca de $60 \%$ de individuos reciben atención en hospitales de la Secretaría de Salud, dependencias que no cuentan con las facilidades para proporcionar DP y que subrogan el servicio de hemodiálisis; por este motivo, los individuos pagan una cuota proporcional a su capacidad de pago.

Por otra parte, se observa que los hospitales de seguridad social atienden a cerca de 32\% de las personas con ERC. Esta cifra está por debajo de lo reportado por García-García y cols. (5), quienes encontraron que en Jalisco, México, 72\% de las personas en TRR reciben atención en hospitales de seguridad social. Esta diferencia está determinada en gran medida por la carencia de aseguramiento en salud, consecuencia del tipo de actividad económica principal en la región, que es la agricultura seguida por el comercio informal. Esto implica que los individuos tengan que ser atendidos por los hospitales de la secretaría de salud y pagar una cuota proporcional a su ingreso mensual (30). Por último, se puede observar que el mayor porcentaje de individuos atendidos en instituciones de seguridad social son los menores de 36 años, lo que lleva a pensar que son ellos quienes acceden con mayor facilidad al campo laboral formal. Cabe destacar que esta comunidad se caracteriza por una alta prevalencia de ERC en personas jóvenes, lo cual difiere con los datos nacionales, donde la media de edad de presentación de la enfermedad se acerca a los 50 años (2). Estos hallazgos sugieren la necesidad de estudiar las características fisiopatológicas de la enfermedad en esta región y los factores de riesgo, tanto individuales, como ambientales, socioculturales y económicos, que pueden estar involucrados en su alta prevalencia en la población joven.

En el presente estudio, $60 \%$ de las personas atendidas en hospitales de la Secretaría de Salud cuenta con los servicios de consulta y hemodiálisis; sin embargo, no se cubren los medicamentos. Este rubro corre a cargo de los individuos y sus familias, lo que en la mayoría de los casos lleva al empobrecimiento. Entre otras, esta es una de las razones por las cuales es probable encontrar poca adherencia, lo que aumenta el riesgo de complicaciones y muerte. El panorama no suele ser mejor para quienes cuentan con servicios de salud, pues la carencia de infraestructura y de personal de salud capacitado limitan la entrega de medicamentos y servicios y encarecen la calidad de la atención (31).

Cualquier sean la condición laboral y del aseguramiento en salud, el enfrentamiento de la enfermedad a nivel físico y psicológico supone un reto para los individuos pues, en la mayoría de los casos, se ven obligados a buscar estrategias para acceder al tratamiento y mejorar sus condiciones de salud. La movilización de recursos individuales, grupales y comunitarios es esencial en este proceso; destaca como recurso inherente al individuo la red de apoyo social, en especial la familiar.

Según los participantes, el apoyo social está presente en forma adecuada, el apoyo emocional es el tipo más frecuente y se correlaciona de manera importante con el empoderamiento. Se observó una correlación positiva muy significativa entre la interacción social positiva y el empoderamiento que, con base a lo establecido por Hall y cols. (11) y por Rosenfield (14), nos hace pensar que el apoyo social positivo podría estar relacionado con la capacidad de los individuos para empoderarse y acceder al tratamiento y ser, a su vez, un determinante del bienestar del individuo como lo reportaron Göz y cols. (32) y Wen y cols. (33). Esta relación deberá ser estudiada con mayor profundidad en el futuro.

Nuestros resultados nos orientan a calificar al apoyo social brindado por la familia como un apoyo negativo que, a pesar de ser fuente de conflicto en un número 
CUADRO 2. Apoyo social y funcionamiento familiar en pacientes con ERCa

\begin{tabular}{|c|c|c|c|c|c|c|c|}
\hline & \multirow{2}{*}{\multicolumn{2}{|c|}{$\begin{array}{c}\text { Media } \pm D E \\
\text { Total }(n=69)\end{array}$}} & \multirow{2}{*}{\multicolumn{2}{|c|}{$\frac{\text { Media } \pm \text { DE }}{\leq 36 \text { años }(n=35)}$}} & \multicolumn{2}{|c|}{ Media $\pm D E$} & \multirow{2}{*}{$P$} \\
\hline & & & & & $>36$ años & $(n=34)$ & \\
\hline Puntaje en la escala de apoyo social & \multicolumn{2}{|c|}{$71,4 \pm 12,8$} & \multicolumn{2}{|c|}{$72,5 \pm 13,6$} & \multicolumn{2}{|c|}{$70,2 \pm 12,0$} & 0,443 \\
\hline Apoyo emocional & \multicolumn{2}{|c|}{$31,8 \pm 7,6$} & \multicolumn{2}{|c|}{$33,0 \pm 7,4$} & \multicolumn{2}{|c|}{$30,5 \pm 7,6$} & 0,18 \\
\hline Apoyo instrumental & \multicolumn{2}{|c|}{$17,6 \pm 3,6$} & \multicolumn{2}{|c|}{$17,6 \pm 3,9$} & \multicolumn{2}{|c|}{$17,6 \pm 3,2$} & 0,969 \\
\hline Apoyo afectivo & \multicolumn{2}{|c|}{$13,3 \pm 2,9$} & \multicolumn{2}{|c|}{$13,1 \pm 3,3$} & \multicolumn{2}{|c|}{$13,5 \pm 2,4$} & 0,589 \\
\hline Interacción social positiva & \multicolumn{2}{|c|}{$17,5 \pm 3,4$} & \multicolumn{2}{|c|}{$17,8 \pm 3,5$} & \multicolumn{2}{|c|}{$17,2 \pm 3,4$} & 0,448 \\
\hline \multicolumn{8}{|c|}{ Puntaje en la escala de funcionamiento familiar } \\
\hline Adaptabilidad & \multicolumn{2}{|c|}{$40,9 \pm 6,3$} & \multicolumn{2}{|c|}{$41,1 \pm 6,5$} & \multicolumn{2}{|c|}{$40,7 \pm 6,1$} & 0,785 \\
\hline \multirow[t]{2}{*}{ Cohesión } & \multicolumn{2}{|c|}{$30,7 \pm 7,6$} & \multicolumn{2}{|c|}{$28,5 \pm 7,1$} & \multicolumn{2}{|c|}{$32,9 \pm 7,6$} & 0,015 \\
\hline & $\mathrm{n}=69$ & $\%$ & $\mathrm{n}=35$ & $\%$ & $\mathrm{n}=34$ & $\%$ & \\
\hline Apoyo global & & & & & & & 0,305 \\
\hline Apoyo escaso & 9 & 13,0 & 6 & 17,1 & 3 & 8,8 & \\
\hline Apoyo suficiente & 60 & 87,0 & 29 & 82,9 & 31 & 91,2 & \\
\hline Apoyo emocional & & & & & & & 0,326 \\
\hline Apoyo escaso & 13 & 18,8 & 5 & 14,3 & 8 & 23,5 & \\
\hline Apoyo suficiente & 56 & 81,2 & 30 & 85,7 & 26 & 76,5 & \\
\hline Apoyo instrumental & & & & & & & 0,97 \\
\hline Apoyo escaso & 6 & 8,7 & 3 & 8,6 & 3 & 8,8 & \\
\hline Apoyo suficiente & 63 & 91,3 & 32 & 91,4 & 31 & 91,2 & \\
\hline Apoyo afectivo & & & & & & & 0,414 \\
\hline Apoyo escaso & 6 & 8,7 & 4 & 11,4 & 42 & 5,6 & \\
\hline Apoyo suficiente & 63 & 91,3 & 31 & 88,6 & 32 & 94,1 & \\
\hline Interacción social positiva & & & & & & & 0,983 \\
\hline Apoyo escaso & 2 & 2,9 & 1 & 2,9 & 1 & 2,9 & \\
\hline Apoyo suficiente & 67 & 97,1 & 34 & 97,1 & 33 & 97,1 & \\
\hline Cohesión familiar & & & & & & & 0,388 \\
\hline Familia no relacionada & 6 & 8,7 & 3 & 8,6 & 3 & 8,8 & \\
\hline Familia semirrelacionada & 25 & 36,2 & 10 & 28,6 & 15 & 44,1 & \\
\hline Familia relacionada & 25 & 36,2 & 16 & 45,7 & 9 & 26,5 & \\
\hline Familia aglutinada & 13 & 18,8 & 6 & 17,1 & 7 & 20,6 & \\
\hline Adaptabilidad familiar & & & & & & & 0,177 \\
\hline Familia rígida & 6 & 8,7 & 5 & 14,3 & 1 & 2,9 & \\
\hline Familia estructurada & 13 & 18,8 & 7 & 20,0 & 6 & 17,7 & \\
\hline Familia flexible & 9 & 13,0 & 6 & 17,1 & 3 & 8,8 & \\
\hline Familia caótica & 41 & 59,4 & 17 & 48,6 & 24 & 70,6 & \\
\hline
\end{tabular}

a Los valores de $P$ para la comparación por grupos de edad se presentan como promedio \pm desviación estándar; los datos categóricos se presentan como frecuencia (porcentaje).

\section{CUADRO 3. Características del empoderamiento por grupo de edad ${ }^{a}$}

\begin{tabular}{|c|c|c|c|c|}
\hline & Total & $\leq 36$ años & $>36$ años & $P$ \\
\hline & $\mathrm{n}=69$ & $\mathrm{n}=35$ & $n=34$ & $r$ \\
\hline Îndice de empoderamiento & $117,5 \pm 14,3$ & $116,0 \pm 16,9$ & $119,1 \pm 11,9$ & 0,385 \\
\hline Empoderamiento individual & $99,9 \pm 11,2$ & $99,9 \pm 2,3$ & $99,8 \pm 8,1$ & 0,982 \\
\hline Empoderamiento comunitario & $17,7 \pm 5,6$ & $16,1 \pm 5,2$ & $19,3 \pm 5,6$ & 0,019 \\
\hline \multicolumn{5}{|c|}{ Dimensiones del empoderamiento individual } \\
\hline Poder para ejercer control & $28,2 \pm 3,5$ & $27,8 \pm 3,6$ & $28,8 \pm 3,3$ & 0,199 \\
\hline Poder para la elección & $35,8 \pm 5,4$ & $34,9 \pm 4,5$ & $36,6 \pm 6,1$ & 0,176 \\
\hline Poder para el cambio & $19,7 \pm 3,1$ & $19,3 \pm 3,2$ & $20,0 \pm 3,0$ & 0,268 \\
\hline Locus de control & $17,9 \pm 3,8$ & $16,9 \pm 3,3$ & $19,0 \pm 4,1$ & 0,026 \\
\hline
\end{tabular}

a Los valores de $P$ para la comparación por grupos de edad se presentan como promedio \pm desviación estándar.

elevado de ocasiones, puede impulsar a buscar otras fuentes de apoyo social. Este hallazgo está estrechamente ligado con la correlación encontrada entre interacción social positiva y empoderamiento y concuerda con una de las teorías de empoderamiento psicológico más importantes que es la de Zimmerman (10, 34), quien
CUADRO 4. Correlaciones entre empoderamiento total y categorías de apoyo social y funcionamiento familiar

\begin{tabular}{lcc}
\hline Variable & Coeficiente & $P$ \\
\hline Apoyo social global & 0,2593 & 0,0314 \\
Apoyo emocional & 0,2448 & 0,0426 \\
Apoyo instrumental & $-0,0011$ & 0,9926 \\
Apoyo afectivo & 0,1986 & 0,1019 \\
Interacción social positiva & 0,3326 & 0,0052 \\
Adaptabilidad & 0,2308 & 0,0564 \\
Cohesión & 0,1931 & 0,112 \\
\hline
\end{tabular}

apunta que uno de los componentes fundamentales del empoderamiento es el interactivo.

Dadas las características de la población y en acuerdo con nuestros resultados, concluimos que el apoyo social es una herramienta clave para el empoderamiento. La evidencia científica que precede a este trabajo nos permite plantear que la presencia de ambos constructos en niveles adecuados constituye un elemento clave para enfrentar las vicisitudes de la ERC. Por otra parte, sugerimos que tanto el apoyo social como el empoderamiento forman parte de la maquinaria indispensable para controlar los recursos disponibles, acceder a los sistemas de salud y participar de manera activa y junto con el personal de salud en la toma de decisiones, sobre todo en contextos caracterizados por inequidades pronunciadas.

Una de las limitaciones más importantes de nuestro estudio es que la muestra elegida no permite hacer generalizaciones, pues la extracción de la muestra de una población que por su naturaleza posee características propias del empoderamiento, la hace diferente al resto de pacientes con ERC. Por otra parte, mediante el acercamiento elegido no es posible identificar elementos como las fuentes y mecanismos de obtención de la información, los procesos de análisis de la esta con respecto a la realidad individual y social, la toma de decisiones conscientes, si el empoderamiento ha transformado estructuras de poder resultando en la mejora de las oportunidades de atención a la ERC a nivel individual y en toda la población, y los mecanismos de participación de la red de apoyo social en estos procesos. Además, el diseño dificulta el estudio de la direccionalidad de la asociación existente entre el apoyo social y el empoderamiento.

Con el objetivo de conocer más a fondo el fenómeno del empoderamiento, los mecanismos de acción de la red de 
apoyo y su impacto social, se sugiere que en futuras investigaciones se aborde el problema desde una perspectiva fenomenológica, mediante metodologías cualitativas que permitan indagar las vivencias y experiencias de los individuos.

Conflicto de intereses. Ningun declarado por los autores.

\section{REFERENCIAS}

1. Hill NR, Fatoba ST, Oke JL, Hirst JA, O'Callaghan CA, Lasserson DS, et al. Global prevalence of chronic kidney disease: a systematic review and meta-analysis. PLoS ONE. 2016;11(7).

2. Obrador GT, García-García G, Villa AR, Rubilar X, Olvera N, Ferreira E, et al. Prevalence of chronic kidney disease in the Kidney Early Evaluation Program (KEEP) México and comparison with KEEP US. Kidney Int. 2010;77(S116):S2-S8.

3. Amato D, Álvarez-Aguilar C, CastanedaLimones R, Rodríguez E, Ávila-Díaz M, Arreola F, et al. Prevalence of chronic kidney disease in an urban Mexican population. Kidney Int. 2005;97:S11-S7.

4. Franco-Marina F, Tirado-Gómez LL, Venado Estrada A, Moreno-López JA, Pacheco-Domínguez RL, Durán-Arenas L, et al. Una estimación indirecta de las desigualdades actuales y futuras en la frecuencia de la enfermedad renal crónica terminal en México. Salud Publica México. 2011;53(S4):S506-S15.

5. García-García G, Briseño-Renteria G, Luquin-Arellan VH, Gao Z, Gill J, Tonelli $M$. Survival among patients with kidney failure in Jalisco, Mexico. J Am Soc Nephrol. 2007;18:1922-7.

6. Wingard R. Reducing Early Mortality in Patients on Dialysis: Lessons from the RightStart Program. Nephrol Nurs J. 2009; 36(2):215-20.

7. Thong M, Kaptein AA, Krediet RT, Boeschoten EW, Dekker FW, Group ftNCSotAoDNS. Social support predicts survival in dialysis patients. Nephrol Dial Transpl. 2007;22:845-50.

8. Kabeer N. Between affiliation and autonomy: navigating pathways of women's empowerment and gender justice in rural Bangladesh. Dev Change. 2011;42(2):499-528.

9. Aujoulat I, d'Hoore W, Deccache A. Patient empowerment in theory and practice: Polysemy or cacophony? Patient Educ Couns. 2007;66:13-20.

10. Perkins D, Zimmerman M. Empowerment theory, research, and application. Am J Commun Psychol. 1995;23(5):569-79.

11. Hall GB, Nelson G. Social networks, social support, personal empowerment, and the adaptation of psychiatric consumers/survivors: Path analytic models. Soc Sci Med. 1996;43(12):1743-54

12. DiMatteo MR. Social support and patient adherence to medical treatment: a meta-analysis. Health Psychol. 2004;23(2): 207-18.

13. Oftedal B, Bru E, Karlsen B. Social support as a motivator of self-management among adults with type 2 diabetes. Journal of Nursing and Healthcare of Chronic Illness. 2011;3:12-22.

14. Rosenfield S. Factors Contributing to the Subjective Quality of Life of the Chronic Mentally Ill. Journal of Health and Social Behavior. 1992;33(4):299-315.

15. Pick S, Sirkin J, Ortega I, Osorio P, Martínez $\mathrm{R}$, Xocolotzin U, et al. Escala Para Medir Agencia Personal y Empoderamiento (ESAGE). Rev Interam Psicol. 2007;41(3): 259-304.

16. Sherbourne CD, Stewart AL. The MOS Social Support Survey. Social Science \& Medicine. 1991;32(6):705-14.

17. Rodríguez S, Enrique HC. Validación Argentina del Cuestionario MOS de Apoyo Social Percibido. Psicodebate. 2007; 7:155-68.

18. Londoño NH, Henao GC, Puerta IC, Posada S, Arango D, Aguirre-Acevedo DC. Propiedades psicométricas y validación de la escala de estrategias de coping modificada (EEC-M) en una muestra colombiana. Revista Universitas Psychologicas. 2006;5(2):327-49.

19. Gómez CFJ, Irigoyen CA, Ponce RER, Mazón RJJ, Dickinson BME, Sánchez GME, et al. Versión al español y adaptación transcultural de FACES III (Family Adaptability and Cohesión Evaluation Scales). Archivos de Medicina Familiar. 1999;1:73-9.

20. Ponce-Rosas ER, Gómez-Clavelinab FJ, Terán-Trilloc M, Irigoyen-Coriad AE, Landgrave-Ibáñeze S. Validez de constructo del cuestionario FACES III en español (México). Atención Primaria. 2002;30(10): 624-30.

21. McMillan DW. Sense of community, theory not a value: a response to Nowell and Boyd. Journal of Community Psychology. 2011;39:507-19.

22. McMillan DW, Florin P, Stevenson J, Kerman B, Mitchell RE. Empowerment praxis in community coalitions. American Journal of Community Psychology. 1995; 23:699-727.

23. Narayan D. Empowerment and poverty reduction: a sourcebook. Washington: World Bank; 2002

24. Ochman-Ikanowicz MB. Políticas sociales y empoderamiento de las mujeres. Una promesa incumplida. Estudios Políticos. 2016;48:32-51.

25. Arredondo A, Luna G, Aldaz V, Orozco E. Equidad e inequidades en salud en México: niveles, tendencias y evidencias en el contexto de la reforma. Salud en Tabasco. 2006:415-24.
Declaración. Las opiniones expresadas en este manuscrito son responsabilidad del autor y no reflejan necesariamente los criterios ni la política de la RPSP/PAJPH y/o de la OPS.
26. Barraza-Lloréns M, Panopoulou G, Díaz BY. Income-related inequalities and inequities in health and health care utilization. Rev Panam Salud Publica. 2013;33(2):122-30.

27. Juárez-Ramírez C, Márquez-Serrano $M$, Salgado de Snyder N, Pelcastre-Villafuerte BE, Ruelas-González MG, Reyes-Morales H. La desigualdad en salud de grupos vulnerables de México: adultos mayores, indígenas y migrantes. Rev Panam Salud Publica. 2014;35(4):284-90.

28. Méndez-Durán A, Ignorosa-Luna $\mathrm{MH}$, Pérez-Aguilar G, Rivera-Rodríguez FJ, González-Izquierdo JJ, Dávila-Torres J. Estado actual de las terapias sustitutivas de la función renal en el Instituto Mexicano del Seguro Social. Revista Médica del Instituto Mexicano del Seguro Social. 2016;54(5):588-93.

29. Cusumano AM, Garcia-Garcia G, Gonzalez-Bedat MC, Marinovich S, Lugon J, Poblete-Badal $\mathrm{H}$, et al. Latin American dialysis and transplant registry: 2008 prevalence and incidence of end-stage renal disease. Kidney Int Suppl. 2013;3:153-6.

30. Gómez-Dantés O, Sesma S, Becerril VM, Knaul FM, Arreola H, Frenk J. Sistema de salud de México. Salud Publica Mexico. 2011;53(S2):S220.S32.

31. Hamui SA, Fuentes GR, Aguirre HR Ramírez ROF. Expectativas y experiencias de los usuarios del Sistema de Salud en México: un estudio de satisfacción con la atención médica. México: Facultad de Medicina, Universidad Autónoma de México. CCINSHRAE; 2012.

32. Göz F, Karaoz S, Goz M, Ekiz S, Cetin I. Effects of the diabetic patients' perceived social support on their quality-of-life. J Clin Nurs. 2007;16(7):1353-60.

33. Wen LK, Shepherd MD, Parchman ML. Family support, diet, and exercise among older Mexican Americans with type 2 diabetes. The Diabetes educator. 2004;30(6): 980-93.

34. Zimmerman MA. Further explorations in empowerment theory: An empirical analysis of psychological empowerment. Am J Commun Psychol. 1992;20(6): 707-27.

Manuscrito recibido el 28 de mayo de 2017. Aceptado para publicación, tras revisión, el 14 de agosto de 2017. 
ABSTRACT Objective. Identify the degree of empowerment and the characteristics of the social support for individuals with chronic kidney disease (CKD).

Methods. Cross-sectional study of people with chronic kidney disease living in the

\section{Empowerment and social support for patients with chronic kidney disease: case study in Michoacán, Mexico}

Keywords Power; social support; renal insufficiency, chronic.
RESUMO Objetivo. Identificar o nível de empoderamento e apoio social de pacientes com nefropatia crônica.

Métodos. Estudo transversal realizado com pacientes com nefropatia crônica residentes no município de Fidalgo, Michoacán, México, que participavam de uma associação civil de pacientes renais crônicos (Asociación de Enfermos del Riñón). Foram investigados aspectos pessoais e sociodemográficos dos pacientes associados à doença, nível de empoderamento e apoio social e funcionamento familiar.

Resultados. Cerca de $90 \%$ da amostra estudada dispõem de uma rede de apoio social satisfatória e $40 \%$ pertencem a famílias com boa coesão ou coesão média. A pontuação global de empoderamento foi de 117,5 \pm 14,3 e o empoderamento comunitário foi maior no grupo com idade mais avançada $(P<0,05)$. Interação social positiva é o componente da rede de apoio social com correlação mais forte com o nível de empoderamento $(\mathrm{r}=0,333 ; P<0,01)$.

Conclusões. O empoderamento é determinado pela rede de apoio social, sendo também um determinante deste apoio, e facilita o acesso à terapia renal substitutiva nesta comunidade.

Palavras-chave Poder; apoio social; insuficiência renal crónica. 